\title{
Longitudinal analysis of the capacities of community health workers mobilized for seasonal malaria chemoprevention in Burkina Faso
}

\author{
Abel Bicaba, Luc Serme ${ }^{1}$, Gaël Chetaille², Gountante Kombate ${ }^{1}$, Alice Bila ${ }^{1}$ and Slim Haddad² ${ }^{2 *}$
}

\begin{abstract}
Background: Seasonal malaria chemoprevention (SMC) relies on community health workers to distribute drugs. This study assessed: (1) the capacity of community-based distributors (CBDs) at the start and end of a campaign and from one campaign to another after training or refresher courses before each round; (2) to what extent CBDs' experience over several campaigns contributed to measurable increase in their capacities; and (3) to what extent the training and experience of committed CBDs helped the less productive to catch up.
\end{abstract}

Methods: A longitudinal analysis was conducted in one Burkina Faso health district during the 2017 and 2018 campaigns. A panel including all CBDs was created. Their capacities were observed after: (1) initial training for the 2017 season; (2) refresher training for that year's fourth round; and (3) initial training for the 2018 season. All were invited to complete a questionnaire at the end of training with 27 multiple-choice questions on their main tasks. Observers noted content coverage and conditions under which training sessions were conducted.

Results: The 612 CBDs showed, on average, high understanding of their tasks from the start of the annual campaigns. Tasks related to communicating with parents and reporting were best mastered. Their capacities grew from round to round and campaign to campaign, after most had undergone training and been supervised by head nurses. The greatest progress was in the technical components, considered more complex, which involved selecting eligible children, choosing the correct drug packet, and referring children to health professionals. Retaining CBDs from one round to the next benefited everyone, whatever their starting level. Groups that initially obtained the lowest scores (women, illiterates, youngest/oldest) progressed the most.

Conclusion: These results confirm the potential of using CBDs under routine programme implementation. Mandating CBDs with targeted tasks is a functional model, as they achieve mastery in this context where investments are made in training and supervision. Losing this specificity by extending CBDs' mandates beyond SMC could have undesirable consequences. The added value of retaining committed CBDs is high. It is suggested that motivation and commitment be considered in recruitment, and that a supportive climate be created to foster retention.

Keywords: Seasonal malaria chemoprevention, Community health workers' performance, Training, Evaluation

*Correspondence: Slim.Haddad@fmed.ulaval.ca

${ }^{2}$ Centre de Recherche du Centre Hospitalier Universitaire de I'Université Laval, Quebec, Canada

Full list of author information is available at the end of the article

\section{Background}

As of 2017, 12 Sahelian countries had integrated seasonal malaria chemoprevention (SMC) into their national malaria prevention and control programmes (NMPCP), 
covering slightly more than half of the 29.3 million eligible children [1]. The implementation of SMC activities tends to follow the World Health Organization (WHO) policy recommendation on SMC for Plasmodium falciparum malaria control in highly seasonal transmission areas [2]. Preventive anti-malarial drugs are provided to eligible children during the peak of malaria transmission. The treatment is administered for three successive days and then repeated monthly, for a total of three or four rounds per campaign. The provision of SMC through a door-to-door strategy relies to a large extent on community health workers, called community-based distributors (CBDs). CBDs receive an allowance of approximately US $\$ 10$ per day. Their mandate typically includes sensitizing the population before each round and administering day-one doses to eligible children. CBDs also have to provide parents with second- and third-day doses and report on their own activity.

There is growing evidence that SMC is effective in real-world conditions [3-5]. These results support the idea that the overall strategy and, more specifically, the involvement of CBDs, is effective and efficient [6]. However, research on CBDs' performance has been quite limited to date, and little is known about their ability to properly accomplish the whole set of technical, relational, and recording/notification tasks assigned to them. Training and supervision are often identified as major contributors to CBDs' knowledge, skills, and motivation [7-12]. It has nonetheless been suggested that more evidence is needed about effective approaches to training $[8,10,12]$, including in malaria programmes [13].

This paper explores CBDs' capacities in Burkina Faso. SMC was initiated in that country in 2014. Each campaign targets children from 3 to 59 months of age, and consists of four monthly rounds (July to October). Delivery is mostly door-to-door. SMC was scaled up rapidly and expanded into the 70 health districts of the country in 2019. More than 40,000 $(41,183)$ CBDs were hired and trained to cover 3.6 million targeted children.

This paper presents the results of a longitudinal analysis conducted in one health district in Burkina Faso during the 2017 and 2018 campaigns. First, the study assessed and compared CBDs' capacities to perform the tasks assigned to them at the beginning and end of a campaign, between the first and fourth rounds, and then from one campaign to the next, at the time of the first round. The second objective was to assess the increase in CBDs' capacities and the added value of districts' efforts to retain them and cultivate their commitment. The hypothesis was that the experience acquired by these CBDs would result in a measurable increase in their capacities over time. A final objective was to verify to what extent the successive training sessions and experience acquired by these committed CBDs helped the less productive to catch up with their peers.

\section{Methods \\ Design}

The study covered a health district in the country's central health region. Malaria is meso-endemic and transmission is seasonal; the rainy season lasts 4 to 6 months. A panel was created that included all CBDs in the district. The CBDs' capacities were observed at three times: (1) in July 2017, after the initial training for the campaign; (2) at the fourth round of the same year, i.e., 3 months later, after the refresher training; and (3) in July 2018, after the annual initial training. The panel was open populationbased, including all CBDs in service during one or more of the three observation periods.

\section{Setting}

CBDs were trained all over the country following a cascade model based on WHO [2]. The cascade was initiated by the NMPCP and went through regional and district officers, and then head nurses of primary healthcare centres. All CBDs of the country attended the training, usually provided in primary healthcare centres. A fullday initial training was offered just before the start of the campaign, and then before the second, third, and fourth rounds. Training sessions were standardized based on teaching material provided by the NMPCP. The training included presentations, demonstrations, and simulations covering nine skill sets: (1) being able to justify and explain the reasons for administering SMC; (2) knowing what to do upon arrival in the household; (3) identifying and selecting eligible children and those who should be referred to the health centre; (4) selecting and administering the packet of medicine appropriate to the child's age; (5) monitoring the child after the medicine is taken and acting appropriately in case of complications; (6) providing parents with second-and third-day doses and showing them the steps they have to take to administer them; (7) instructing parents on how to monitor treated children and what to do if complications arise; (8) providing information on how to prevent malaria and how to obtain care as soon as possible when malaria symptoms appear; and (9) carrying out notification, reporting, and concession-marking activities, i.e., marking compound walls to indicate the household was visited.

\section{Data collection}

Fifty surveyors were trained and then distributed among the various health centres on the day of each training. An observation grid for the training process was used to assess, in the first place, the trainers' coverage of the content to be delivered (what is SMC; criteria for selecting 
eligible children; steps of SMC; communication with parents; referral; side effects). A second component of the grid dealt with the conditions in which the training was provided, the interactions between learners and trainers, the dynamism of the trainer, the working environment, and the duration of the session. The grid consisted of 44 items. At each round, two to three observers per site completed the grid and then developed a common grid by consensus.

All CBDs were invited to complete a questionnaire at the end of the training. It included 27 multiple-choice questions, each focused on a specific task. They were asked about their gender, age, education, previous experience as a community health worker, and participation in previous campaigns. The questionnaire was administered face-to-face in the local language; each interview lasted approximately $20 \mathrm{~min}$. The questionnaire was tested and revised beforehand.

\section{Analyses}

Seven scores were constructed to assess the completeness of the training content. Each score corresponded to a group of tasks defined in the training (explanation of the SMC; criteria for selecting children; administration of medication; communication with parents; referral; side effects). Nine intermediate scores and a total score were constructed to assess the CBDs' capacities in each round. The scores were transposed to a $0-100$ scale to facilitate comparisons. All analyses were performed using Stata $\odot$ 15. Multilevel models adapted to the longitudinal and the hierarchical data structure (each training site being a cluster of CBDs) were used [14]. Regressions were based on censored tobit models when scores were transformed in a $0-100$ censored range of values ("metobit" mixedeffects routine in STATA $\odot$ ). The progression of CBDs' capacities was assessed based on the overall skills score for those who had participated in three rounds. Double difference was used to assess the effects of each round by CBDs' gender, education, and age.

\section{Results}

The sample consisted of 23 health centres in the first and second rounds; a 24th centre was opened in 2018. No trainer or CBD refused to participate. The number of CBDs varied slightly from one round to another, with head nurses adjusting their staffing based on the CBDs' workloads and availabilities (panel participation pattern in Additional file 1). About half of the 612 CBDs participated in the three rounds (47\%). They included mainly farmers, men, and people with a primary school education who had participated in at least one previous campaign (Table 1). Two-thirds had previously worked as community health workers for other activities.
Table 1 Characteristics of CBDs in the first round of the survey (P1-2017)

\begin{tabular}{|c|c|c|}
\hline Characteristics & $\mathbf{n}$ & Mean or \% \\
\hline Age & 424 & 32.3 \\
\hline \multicolumn{3}{|l|}{ Sex } \\
\hline Male & 305 & 72.0 \\
\hline Female & 119 & 28.0 \\
\hline \multicolumn{3}{|l|}{ Living in the distribution village } \\
\hline Yes & 341 & 80.4 \\
\hline No & 83 & 19.6 \\
\hline \multicolumn{3}{|l|}{ Education level } \\
\hline No schooling & 23 & 50.4 \\
\hline Literate & 69 & 16.3 \\
\hline Primary school not completed & 43 & 10.1 \\
\hline Primary school completed & 289 & 68.2 \\
\hline \multicolumn{3}{|l|}{ Main activity } \\
\hline Agriculture & 284 & 67.1 \\
\hline Livestock & 15 & 3.6 \\
\hline Commerce & 20 & 4.7 \\
\hline Studies & 82 & 19.4 \\
\hline Other & 22 & 5.2 \\
\hline \multicolumn{3}{|l|}{ Years of experience as a CHW } \\
\hline None & 159 & 37.5 \\
\hline 1 year or less & 90 & 21.2 \\
\hline 2 years & 37 & 8.7 \\
\hline 3 years or more & 138 & 32.6 \\
\hline \multicolumn{3}{|c|}{$\begin{array}{l}\text { Number of SMC campaigns in which the } \\
\text { CBD has already participated }\end{array}$} \\
\hline None & 55 & 13.0 \\
\hline One & 132 & 31.1 \\
\hline Two or more & 237 & 55.9 \\
\hline
\end{tabular}

Observation showed that the intended contents were covered in the training sessions (Table 2). Content coverage decreased, however, between the first and fourth rounds. Thus, it appeared less attention was given to refresher training, even though the national curriculum directives call for trainers to provide it in the same way. The difference was significant for overall completeness $\left(\Delta=-11.4 \% ; \mathrm{CI}_{95}=[-18.1 \%:-4.7 \%]\right)$, SMC explanations $\left(\Delta=-0.30 ; \mathrm{CI}_{95}=[-0.76:-0.03]\right)$, tasks to be performed at each step $\left(\Delta=-1.74 ; \mathrm{CI}_{95}=[-2.63\right.$ : $-0.85])$, and communication with parents $(\Delta=-0.39$; $\left.\mathrm{CI}_{95}=[-0.77:-0.01]\right)$. This progression was accompanied by a halving of the duration of the training session $\left(\Delta=-114 \mathrm{mn} ; \mathrm{CI}_{95}=[-167:-62]\right)$. The comparison of the initial training sessions for the two consecutive campaigns showed no difference in the content actually covered. Finally, the conditions under which the training was conducted varied little from one period to another. 
Table 2 Completeness of content and conduct of training at each round

\begin{tabular}{|c|c|c|c|c|c|c|c|c|c|}
\hline \multirow[t]{3}{*}{ Score } & & \multirow{3}{*}{$\begin{array}{l}\text { Number } \\
\text { of items }\end{array}$} & \multicolumn{3}{|l|}{ Round } & \multicolumn{2}{|l|}{ Variation } & \multicolumn{2}{|c|}{ Relative variation } \\
\hline & & & P1 2017 & P4 2017 & P1 2018 & $\mathrm{P} 1 \rightarrow \mathrm{P} 4$ & $\mathrm{P} 1 \rightarrow \mathrm{P} 1$ & P1-P4 & P1-P1 \\
\hline & & & (1) & $(2)$ & (3) & $(2)-(1)^{*}$ & $(3)-(1)^{*}$ & $(2)-(1) /(1)$ & $(3)-(1) /(1)$ \\
\hline \multicolumn{10}{|c|}{ Training content } \\
\hline C1 & SMC well explained & 6 & 5.9 & 5.5 & 6.0 & -0.39 & 0.09 & $-7 \%$ & $1 \%$ \\
\hline$C 2$ & Selection criteria covered & 14 & 13.2 & 12.4 & 13.7 & -0.74 & 0.52 & $-6 \%$ & $4 \%$ \\
\hline C3 & Steps of SMC covered & 13 & 12.2 & 10.5 & 12.1 & -1.74 & -0.13 & $-14 \%$ & $-1 \%$ \\
\hline C4 & Communication covered & 4 & 3.9 & 3.5 & 3.9 & -0.39 & -0.04 & $-10 \%$ & $-1 \%$ \\
\hline C5 & Referral covered & 4 & 4.0 & 3.7 & 4.0 & -0.26 & 0.00 & $-7 \%$ & $0 \%$ \\
\hline \multirow[t]{2}{*}{ C6 } & Side effects covered & 2 & 1.8 & 1.3 & 1.7 & -0.52 & -0.04 & $-29 \%$ & $-2 \%$ \\
\hline & $\begin{array}{l}\text { Completeness score trans- } \\
\text { formed (range: 0-100) }\end{array}$ & 43 & $97.7 \%$ & $86.25 \%$ & $99.7 \%$ & $11.4 \%$ & $2.0 \%$ & & \\
\hline \multicolumn{10}{|c|}{ Conduct of the training } \\
\hline D1 & General conditions & 4 & 2.3 & 2.2 & 2.9 & -0.1 & 0.6 & $-6 \%$ & $24 \%$ \\
\hline D2 & Dynamism of the trainer & 3 & 2.5 & 2.5 & 2.7 & 0.0 & 0.3 & $0 \%$ & $11 \%$ \\
\hline D3 & Trainer-participant interactions & 5 & 5.0 & 4.5 & 5.0 & -0.5 & 0.0 & $-10 \%$ & $0 \%$ \\
\hline \multirow[t]{2}{*}{ D4 } & Work environment & 3 & 1.9 & 1.7 & 2.0 & -0.1 & 0.1 & $-7 \%$ & $7 \%$ \\
\hline & $\begin{array}{l}\text { Conduct score transformed } \\
\text { (range } 0-100)\end{array}$ & 15 & $77 \%$ & $73 \%$ & $84 \%$ & $-3.2 \%$ & $7.9 \%$ & & \\
\hline \multicolumn{10}{|c|}{ Duration of the training (min.) } \\
\hline Mean & & & 229 & 115 & 203 & -114 & -27 & $-50 \%$ & $-12 \%$ \\
\hline Median & & & 195 & 133 & 202 & & & & \\
\hline Minimum & & & 24 & 35 & 45 & & & & \\
\hline Maximum & & & 470 & 256 & 414 & & & & \\
\hline
\end{tabular}

* Crude comparisons, not adjusted. In italics, significant differences between rounds $(p<0.05)$

On average, the CBDs demonstrated a high level of understanding of their tasks (Table 3 and Additional file 2). All else being equal, the highest scores were obtained by literate CBDs, those in the intermediate age group (23-39 years), and those who had previously participated in SMC campaigns (Table 4). Previous experience as a community health worker did not seem to be an added value for the SMC programme.

On average, it was in the fourth round that CBDs' know-how was highest, after the majority had gone

Table 3 Mean capacities of CBDs at each of the three observation rounds

\begin{tabular}{|c|c|c|c|c|c|c|c|}
\hline \multicolumn{2}{|c|}{ Knowledge score } & \multirow{3}{*}{$\begin{array}{l}\text { Number } \\
\text { of items }\end{array}$} & \multicolumn{3}{|c|}{ Scores by round (\%) } & \multicolumn{2}{|c|}{ Variation* } \\
\hline & & & \multirow{2}{*}{$\begin{array}{l}\text { P1 } 2017 \\
\text { (1) }\end{array}$} & & & & \\
\hline & & & & $\begin{array}{l}\text { P4 } 2017 \\
\text { (2) }\end{array}$ & $\begin{array}{l}\text { P1 } 2018 \\
\text { (3) }\end{array}$ & $\begin{array}{l}\text { P1->P4 } \\
(2)-(1)\end{array}$ & $\begin{array}{l}\text { P1->P1 } \\
\text { (3)-(1) }\end{array}$ \\
\hline S1 & Reasons for SMC & 1 & $90.3 \%$ & $96.4 \%$ & $93.8 \%$ & $+06.1 \%$ & $+03.5 \%$ \\
\hline S2 & What to do upon arrival in the household & 3 & $96 \%$ & $98 \%$ & $98 \%$ & $+01.5 \%$ & $+01.6 \%$ \\
\hline S3 & Selection of eligible children & 5 & $82 \%$ & $87 \%$ & $85 \%$ & $+04.4 \%$ & $+02.3 \%$ \\
\hline S4 & When to refer the child & 3 & $78 \%$ & $86 \%$ & $71 \%$ & $+07.7 \%$ & $-06.8 \%$ \\
\hline S5 & Selection of drug packet & 5 & $91 \%$ & $97 \%$ & $97 \%$ & $+06.2 \%$ & $+05.6 \%$ \\
\hline S6 & Immediate monitoring of child & 3 & $86 \%$ & $87 \%$ & $94 \%$ & $+00.7 \%$ & $+07.8 \%$ \\
\hline S7 & Subsequent monitoring by parents & 3 & $90 \%$ & $93 \%$ & $93 \%$ & $+02.9 \%$ & $+03.1 \%$ \\
\hline S8 & Advising parents & 3 & $100 \%$ & $100 \%$ & $100 \%$ & $-00.1 \%$ & $-00.2 \%$ \\
\hline S9 & Noting the concession & 1 & $96 \%$ & $98 \%$ & $88 \%$ & $+02.1 \%$ & $-07.7 \%$ \\
\hline Total & Overall know-how & 27 & $89 \%$ & $93 \%$ & $91 \%$ & $+03.8 \%$ & $+02.0 \%$ \\
\hline
\end{tabular}

* Crude comparisons, not adjusted. In italics, significant differences between rounds $(p<0.05)$ 
Table 4 Characteristics of CBDs associated with their know-how

\begin{tabular}{|c|c|c|c|}
\hline \multirow[t]{3}{*}{ Characteristics of CBDs } & \multicolumn{3}{|c|}{ Mean score (\%) } \\
\hline & \multirow[t]{2}{*}{ Crude (\%) ${ }^{a}$} & \multicolumn{2}{|l|}{ Adjusted $^{\mathbf{b}}$} \\
\hline & & Estimates (\%) & Difference (\%) \\
\hline \multicolumn{4}{|l|}{ Age } \\
\hline Q1 (<23) & 89.7 & 90.2 & \\
\hline Q23 (23-39) & 91.6 & 91.9 & +01.6 \\
\hline Q4 (> 40) & 90.4 & 91.0 & +00.8 \\
\hline \multicolumn{4}{|l|}{ Sex } \\
\hline Male & 91.1 & 91.5 & \\
\hline Female & 90.3 & 90.6 & -00.9 \\
\hline \multicolumn{4}{|l|}{ Education } \\
\hline Not educated & 89.4 & 89.1 & \\
\hline Educated & 91.2 & 91.8 & +02.7 \\
\hline \multicolumn{4}{|l|}{ Experience as CHW } \\
\hline None & 90.8 & 91.3 & \\
\hline 1 year & 90.2 & 91.0 & -00.3 \\
\hline 2 years & 91.8 & 91.7 & +00.5 \\
\hline 3 years or more & 90.9 & 91.2 & -00.1 \\
\hline \multicolumn{4}{|c|}{$\begin{array}{l}\text { Participation in previous SMC cam- } \\
\text { paigns }\end{array}$} \\
\hline None & 89.9 & 89.6 & \\
\hline 1 & 90.4 & 91.0 & +01.4 \\
\hline 2 or more & 91.4 & 92.0 & +02.4 \\
\hline 3 or more & 91.1 & 91.7 & +02.1 \\
\hline
\end{tabular}

a Observed values in the sample. Standard errors adjusted for clustering

b Predicted values estimated by tobit models. Standard errors adjusted for clustering

c Differences in expected outcomes. Significant effects in italics ( $\alpha=0.05)$

through the initial training, been supervised by head nurses in the previous rounds, and attended several refresher courses. The most visible changes from one round to the next had to do with the technical components, considered more complex, which involved explaining the SMC, selecting eligible children, choosing the correct packet of medicine to use (there are two models with different colours depending on the child's age), and arranging referrals for children with malaria, fever, or complications requiring professional expertise. The CBDs' average capacities after the initial training for the campaign was higher in the 2018 campaign. However, the gaps noted were slightly smaller than those observed between the beginning and end of the 2017 season. CBDs who had participated in all three rounds of investigation consistently showed higher scores than the others.

Analysis of the panel of CBDs who participated in the three rounds was done to assess the individual progress of perseverant CBDs and compensate for the possible attrition of CBDs who were less motivated or available.
Estimates of the progression of the CBDs' overall skills are presented in Table 5. Between the beginning and end of the 2017 season, estimates suggest an increase of 4.8 points (relative variation of $5.4 \%$ ). The increase was also significant from one campaign to the next. CBD retention appeared to benefit all groups, whether male or female, better educated or less educated, younger or older. Groups of CBDs who were initially less productive also tended to catch up with their more efficient peers. Women made greater progress than men $(+6.3 \mathrm{vs}$. $+4.3)$, both within a campaign and from one campaign to another $(+2.8)$. CBDs with low education levels, who were initially less productive, progressed significantly (+5.4 during the 2017 season and 4.6 from one campaign to the next), partially closing the gap with their more educated peers. The youngest and oldest, less productive at the beginning, also progressed more.

\section{Discussion}

The study revealed, first, that CBDs recruited and trained for SMC showed, on average, a high level of understanding of the tasks they had to perform, right from the start of the annual campaigns. They were most proficient at tasks related to communicating with parents or reporting than tasks related to technical components-selecting eligible children, monitoring children after medicine was taken, or referring children for transfer.

These results are nonetheless consistent with another study on community health workers' performance conducted in Burkina Faso, Nigeria, and Uganda, where 98\% of the tasks were mastered by respondents [15]. They are also consistent with those of a recent observational study conducted in the same context with 14 CBDs, which concluded that they "deliver acceptable quality of care" [16]. Studies have also shown that SMC has contributed significantly to reducing both malaria transmission and the biological prevalence of anaemia in children under five [3]. The results of this study, therefore, support the view that malaria prevention benefits from the involvement of community health workers [17].

CBDs are a particular kind of community health worker, as they are recruited periodically, for only 4 months, and have very focused and specific tasks. As such, they are able to learn relatively quickly and comprehensively. This situation contrasts sharply with those of the "generalist" community health workers in Burkina Faso, whose very broad responsibilities have expanded steadily over the years, and who have been shown not to contribute effectively to the treatment of malaria in children [18]. Many authors have, for several decades, stressed the importance of limiting and concentrating the tasks assigned to community health workers and ensuring they have a reasonable and realistic workload 
Table 5 Factors modifying the progression of CBDs' overall capacities from one round to another (overall score)

\begin{tabular}{|c|c|c|c|c|c|c|c|}
\hline & \multicolumn{3}{|c|}{ Score by round ${ }^{a}$} & \multicolumn{4}{|l|}{ Variation $^{\mathbf{b}}$} \\
\hline & P1-2017 & P4-2017 & P1-2018 & Diff. P4-P1 & Diff. of diff. & Diff. P1-P1 & Diff. of diff. \\
\hline \multirow[t]{2}{*}{ All } & 89.2 & 94.1 & 92.7 & 4.8 & & 3.4 & \\
\hline & {$[88.1,90.4]$} & {$[93.0,95.0]$} & {$[91.4,93.9]$} & {$[3.7,5.9]$} & & {$[2.0,4.8]$} & \\
\hline \multirow[t]{2}{*}{ Male (ref.) } & 89.9 & 94.2 & 92.6 & 4.3 & & 2.7 & \\
\hline & {$[88.7,91.1]$} & {$[93.0,95.4]$} & {$[91.2,94.0]$} & {$[3.1,5.6]$} & 2.0 & {$[1.1,4.3]$} & 2.8 \\
\hline \multirow[t]{2}{*}{ Female } & 87.3 & 93.6 & 92.9 & 6.3 & {$[-0.6,4.5]$} & 5.5 & {$[0.2,5.4]$} \\
\hline & {$[85.6,89.1]$} & {$[91.8,95.5]$} & {$[91.1,94.6]$} & {$[4.1,8.5]$} & & {$[3.3,7.8]$} & \\
\hline \multirow[t]{2}{*}{ No schooling (ref.) } & 86.2 & 91.7 & 90.8 & 5.4 & & 4.64 & \\
\hline & {$[84.4,88.1]$} & {$[89.8,93.6]$} & {$[88.8,92.8]$} & {$[3.1,7.8]$} & -0.8 & {$[3.3,5.9]$} & -1.5 \\
\hline \multirow[t]{2}{*}{ Educated } & 90.2 & 94.9 & 93.3 & 4.5 & {$[-3.6,2.0]$} & 3.05 & {$[-4.2,1.3]$} \\
\hline & {$[89.0,91.4]$} & {$[93.6,96.1]$} & {$[92.0,94.6]$} & {$[2 \cdot 0,7.1]$} & & {$[1.5,4.6]$} & \\
\hline \multirow[t]{2}{*}{ Q1 age (ref) } & 87.4 & 93.7 & 93.2 & 6.3 & & 5.8 & \\
\hline & {$[85.4,89.4]$} & {$[91.7,95.8]$} & {$[91.0,95.5]$} & {$[3.8,8.9]$} & & {$[3.0,8.6]$} & \\
\hline \multirow[t]{2}{*}{ Q23 age } & 90.1 & 94.5 & 93.1 & 4.4 & -2.0 & 3.0 & -2.8 \\
\hline & {$[88.8,91.4]$} & {$[93.1,95.8]$} & {$[91.7,94.5]$} & {$[2.9,5.9]$} & {$[-4.9,0.9]$} & {$[1.3,4.7]$} & {$[-5.9,0.3]$} \\
\hline \multirow[t]{2}{*}{ Q3 age } & 88.8 & 93.6 & 91.4 & 4.8 & -1.5 & 2.7 & -3.1 \\
\hline & {$[87.0,90.6]$} & {$[91.7,95.4]$} & {$[89.6,93.3]$} & {$[2.6,7.0]$} & {$[-5.06,2.0]$} & {$[0.26,5.1]$} & {$[-6.8,0.5]$} \\
\hline
\end{tabular}

a Predicted values estimated by tobit models. Standard errors adjusted for clustering

b Differences in expected outcome and differences in differences. Significant effects in italics $(a=0.05)$

[8, 19-22]. There is, therefore, legitimate concern about the decision taken by the national authorities and their partners to extend the CBDs' mandate to include malnutrition screening as of the second round of the 2018 campaign. This added task was maintained in the CBDs' activity package in 2019 .

Second, the study seems to us to highlight the considerable advantage of fostering commitment among CBDs and retaining them from one campaign to another. On one hand, CBDs who have participated in several campaigns have a better understanding of their tasks. On the other, the capacities of the same CBD increase significantly over the course of a campaign and from one campaign start to the next. The progress is significant even though the baselines are already quite high and margins for progress are naturally limited. Research suggests that the capacities of community health workers tend to wane rather quickly $[11,23,24]$ and that refresher training effectively enhances their performance [12, 25, 26]. The CBDs of the 2017 and 2018 campaigns thus seemed to have benefited from the cumulative contribution of each initial campaign training session, the supervision provided by the health teams during each round, and the regular refresher training preceding each round. These repeated exposures helped particularly to remedy their initial weaknesses in the technical spheres, which they had more difficulty grasping.

Third, the retention of CBDs benefited everyone, whether or not those CBDs were initially productive. It was, however, of greater benefit to those who, because of their limited education, age, or gender, had a poorer initial understanding of their tasks. Women completely closed the gap with their male counterparts, and the gaps were narrowing between uneducated CBDs and those with higher levels of education, as well as between the youngest/oldest and the others. This is the first study to examine such changes among community health workers.

Many studies have shown that the personal characteristics of community health workers can be associated with their performance $[8,11,15,27]$, suggesting that the CBDs to be trained should be carefully selected [11, 28]. However, recent reviews reveal that results, particularly with regard to performance compared by type and sex, are not always consistent [12], and that "studies of workers with lower baseline performance showed greater improvements in prescribing medications..., vaccinating children,... and counseling..." [29]. As well, the more educated CBDs, who are traditionally more productive, are also less committed and more likely to drop out $[8,11$, $15,27]$. The cumulative effect of experience and repeated exposures to training-supervision dyads has reduced or closed the initial gap in understanding, including for the less educated. This raises the question of whether it would not be as, or even more, beneficial to include in the CBD selection process criteria relating to their longterm availability and social acceptability, rather than their learning capacities a priori. 
This study appears to us to present the following strengths. It is based on an independent evaluation of an intervention scaled up by national authorities, and not, as is often the case in studies on community health workers, limited to a small-scale experience in selected populations [20]. The fact that the study was conducted on an uncontrolled intervention rather than a pilot or demonstration project reinforces its informative capacity. Using a longitudinal rather than cross-sectional design that covered several rounds and two national campaigns made it possible to evaluate the collective and individual progression of the CBDs' capacities. The risks of selection bias were contained by selecting a district on the basis of its geographical location rather than the anticipated results of the SMC or the motivation of district leaders, as well as by including all available health centre populations and CBDs in that district. The results regarding CBDs' capacity increases are robust and statistically significant, even though the margins for progression were a priori limited by the presence of high baseline values. Finally, the internal validity is strengthened by the convergence of the results and their consistency with studies conducted in the country on household and child coverage, and on the impacts of SMC on malaria transmission.

The study also has limitations. It was conducted in only one district, and it is plausible that there would be natural variations in performance between districts and regions of the country. Evidence of CBDs' good grasp of tasks does not necessarily presume their future performance once in the field, even if it is a prerequisite [30]. Further research would be needed to assess the compliance of CBDs' practices in the field. Nor does the study provide a precise picture of the specific contributions of the initial training, supervision, or refresher sessions to their progression; rather, it provides more general information on the cumulative effects of these actions. Finally, the limited number of training centres does not provide enough power to explore in detail the associations between CBDs' capacities and the quality of training by training centre.

\section{Conclusions}

The study provides new knowledge on a recently introduced intervention modality in the health systems of Sahelian countries. In the context of Burkina Faso, it reports on the capacities of the outreach group of community-based distributors, which is its cornerstone. Three lessons emerge from the experience evaluated. First, CBDs master the tasks assigned to them in this context where efforts are regularly invested in training and mentoring community health workers. The model of CBDs mandated for targeted and specific tasks appears to be more functional and appropriate than the ineffective model of generalist community health workers. The loss of this specificity by extending CBDs' mandates beyond the SMC is potentially hazardous and could jeopardize its effectiveness. Second, CBDs gain greater mastery of the tasks from round to round and campaign to campaign. This progression shows the relevance of a systematic approach to training, supervision, and regular refresher sessions to support routine implementation of the system. Finally, while the increase in CBDs' capacities relates to all community health workers, it is of particular value to those who, because of their limited education, their age, or gender, have a poorer initial understanding of their tasks.

National authorities should focus their efforts on fostering the retention of recruited CBDs and, in the selection processes, take into account their motivation and long-term commitment, rather than focusing primarily on their presumed learning capacities. They would also benefit from cultivating a climate of trust and a spirit of partnership between the CBDs and the health centres, while also strengthening their credibility among the populations.

\section{Supplementary information}

Supplementary information accompanies this paper at https://doi. org/10.1186/s12936-020-03191-y.

Additional file 1. Participation pattern of the panel.

Additional file 2. CBD responses at each round.

\section{Acknowledgements}

The authors would like to extend warm thanks to the health district managers, head nurses, and community-based distributors for their assistance in the field and their participation in the team's work. The authors also thank the national authorities and the National Malaria Control Programme team in charge of the SMC for their availability and the information they provided on SMC imple-

mentation and achievements in Burkina Faso.

\section{Authors' contributions}

$A B$ and $\mathrm{SH}$ designed and coordinated the study and participated in all stages of manuscript writing. $A B, S H, L S, A B, F B$, and $S K$ were involved in developing the method and implementing the study. SH and LS performed the analyses. GC, AB, SH, and LS wrote the first drafts of the paper. All authors read and approved the final manuscript.

\section{Funding}

This research was supported by a Grant (Number Ga-03-2015F) from the Innovating for Maternal and Child Health in Africa Initiative-a partnership between Global Affairs Canada (GAC), the Canadian Institutes of Health Research (CIHR) and Canada's International Development Research Centre (IDRC).

\section{Availability of data and materials}

The datasets used and/or analysed in the current study are available from the corresponding author on reasonable request.

\section{Ethics approval and consent to participate}

The study was approved by the Burkina Faso Health Research Ethics Committee (2016-09-093) and the Ethics Committee of the CHU de Québec Research Centre (F9 - 32471). The study was authorized by the Ministry of Health (No. 
2016 04700/MS/SG/DGS/DLM/PNLP, November 2, 2016) and local health authorities. Written consent from trainers and CBDs was sought at each round. Strict access procedures were applied to ensure the confidentiality of longitudinal data.

\section{Consent for publication}

Not applicable.

\section{Competing interests}

The authors declare they have no financial or non-financial competing interests. This study was an independent research project. The results were presented to the national health authorities, and at no time did the SMC sponsors interfere in the formulation of the research questions, the conduct of the study, or the analysis, interpretation, or publication of the data. The authors declare that they derive no benefit from publishing results that are favourable or unfavourable to SMC.

\section{Author details}

' Société d'Études et de Recherches en Santé Publique, Ouagadougou, Burkina Faso. ${ }^{2}$ Centre de Recherche du Centre Hospitalier Universitaire de I'Université Laval, Quebec, Canada.

Received: 10 October 2019 Accepted: 13 March 2020

Published online: 19 March 2020

\section{References}

1. WHO. World malaria report 2018. Geneva: World Health Organization; 2018.

2. WHO. Seasonal malaria chemoprevention with sulfadoxine-pyrimethamine plus amodiaquine in children: a field guide. Geneva: World Health Organization; 2013.

3. Druetz T, Corneau-Tremblay N, Millogo T, Kouanda S, Ly A, Bicaba A, et al. Impact evaluation of seasonal malaria chemoprevention under routine program implementation: a quasi-experimental study in Burkina Faso. Am J Trop Med Hyg. 2018;98:524-33.

4. Druetz T. Evaluation of direct and indirect effects of seasonal malaria chemoprevention in Mali. Sci Rep. 2018;8:8104.

5. Ntab B, Cissé B, Boulanger D, Sokhna C, Targett G, Lines J, et al. Impact of intermittent preventive anti-malarial treatment on the growth and nutritional status of preschool children in rural Senegal (West Africa). Am J Trop Med Hyg. 2007;77:411-7.

6. Bojang KA, Akor F, Conteh L, Webb E, Bittaye O, Conway DJ, et al. Two strategies for the delivery of IPTC in an area of seasonal malaria transmission in the Gambia: a randomised controlled trial. PLoS Med. 2011;8:e1000409.

7. Viswanathan M, Kraschnewski J, Nishikawa B, Morgan LC, Thieda P, Honeycutt A, et al. Outcomes of community health worker interventions. Evidence report/technology assessment, number 181. Rockville, MD: U.S. Department of Health and Human Services, Agency for Healthcare Research and Quality; 2009.

8. Kok MC, Dieleman M, Taegtmeyer M, Broerse JE, Kane SS, Ormel H, et al. Which intervention design factors influence performance of community health workers in low- and middle-income countries? A systematic review. Health Policy Plan. 2015;30:1207-27.

9. Bosch-Capblanch X, Marceau C. Training, supervision and quality of care in selected integrated community case management (iCCM) programmes: a scoping review of programmatic evidence. J Glob Health. 2014;4:020403.

10. Abdel-All M, Putica B, Praveen D, Abimbola S, Joshi R. Effectiveness of community health worker training programmes for cardiovascular disease management in low-income and middle-income countries: a systematic review. BMJ Open. 2017;7:e015529.

11. Lopes SC, Cabral AJ, de Sousa B. Community health workers: to train or to restrain? A longitudinal survey to assess the impact of training community health workers in the Bolama Region, Guinea-Bissau. Hum Resour Health. 2014;12:8.

12. Scott K, Beckham SW, Gross M, Pariyo G, Rao KD, Cometto G, et al. What do we know about community-based health worker programs? A systematic review of existing reviews on community health workers. Hum Resour Health. 2018;16:39.
13. Wu S, Roychowdhury I, Khan M. Evaluations of training programs to improve human resource capacity for HIV, malaria, and TB control: a systematic scoping review of methods applied and outcomes assessed. Trop Med Health. 2017:45:16.

14. Rabe-Hesketh S, Skrondal A. Multilevel and longitudinal modeling using Stata. College Station: Stata Press; 2005.

15. Siribié M, Ajayi IO, Nsungwa-Sabiiti J, Afonne C, Balyeku A, Falade CO, et al. Training community health workers to manage uncomplicated and severe malaria: experience from 3 rural malaria-endemic areas in sub-Saharan Africa. Clin Infect Dis. 2016;63(suppl 5):S264-9.

16. Kombate G, Guiella G, Baya B, Serme L, Bila A, Haddad S, et al. Analysis of the quality of seasonal malaria chemoprevention provided by community health workers in Boulsa health district, Burkina Faso. BMC Health Serv Res. 2019;19:472.

17. Gilmore B, McAuliffe E. Effectiveness of community health workers delivering preventive interventions for maternal and child health in lowand middle-income countries: a systematic review. BMC Public Health. 2013;13:847.

18. Druetz T, Ridde V, Kouanda S, Ly A, Diabate S, Haddad S. Utilization of community health workers for malaria treatment: results from a three-year panel study in the districts of Kaya and Zorgho, Burkina Faso. Malar J. 2015;14:71.

19. Glenton C, Lewin S, Scheel IB. Still too little qualitative research to shed light on results from reviews of effectiveness trials: a case study of a Cochrane review on the use of lay health workers. Implement Sci. 2011;6:53.

20. Haines A, Sanders D, Lehmann U, Rowe AK, Lawn JE, Jan S, et al. Achieving child survival goals: potential contribution of community health workers. Lancet. 2007;369:2121-31.

21. Simmons R, Koenig MA, Huque AA. Maternal-child health and family planning: user perspectives and service constraints in rural Bangladesh. Stud Fam Plan. 1990;21:187-96.

22. Jaskiewicz W, Tulenko K. Increasing community health worker productivity and effectiveness: a review of the influence of the work environment. Hum Resour Health. 2012;10:38.

23. Armstrong G, Kermode M, Raja S, Suja S, Chandra P, Jorm AF. A mental health training program for community health workers in India: impact on knowledge and attitudes. Int J Ment Health Syst. 2011;5:17.

24. Bhutta ZA, Lassi ZS, Pariyo G, Huicho L. Global experience of community health workers for delivery of health related Millennium Development Goals: a systematic review, country case studies, and recommendations for integration into national health systems. Geneva: World Health Organization and Global Health Workforce Alliance; 2010.

25. Kuule Y, Dobson AE, Woldeyohannes D, Zolfo M, Najjemba R, Edwin BMR, et al. Community health volunteers in primary healthcare in rural Uganda: factors influencing performance. Front Public Health. 2017;5:62.

26. Wanduru P, Tetui M, Tuhebwe D, Ediau M, Okuga M, Nalwadda C, et al. The performance of community health workers in the management of multiple childhood infectious diseases in Lira, northern Uganda - a mixed methods cross-sectional study. Glob Health Action. 2016;9:33194.

27. Bagonza J, Kibira SPS, Rutebemberwa E. Performance of community health workers managing malaria, pneumonia and diarrhoea under the community case management programme in central Uganda: a cross sectional study. Malar J. 2014;13:367.

28. Stekelenburg J, Kyanamina SS, Wolffers I. Poor performance of community health workers in Kalabo District, Zambia. Health Policy. 2002;65:109-18.

29. Nguyen DT, Leung KK, McIntyre L, Ghali WA, Sauve R. Does integrated management of childhood illness (IMCl) training improve the skills of health workers? A systematic review and meta-analysis. PLoS ONE. 2013;8:e66030.

30. Kak N, Burkhalter B, Cooper M-A. Measuring the competence of healthcare providers. Operations Reseach Issue Paper 2(1). Bethesda: Published for the U.S. Agency for International Development (USAID) by the Quality Assurance (QA) Project; 2001.

\section{Publisher's Note}

Springer Nature remains neutral with regard to jurisdictional claims in published maps and institutional affiliations. 